\title{
Obstetric indications for admission to the intensive care unit of a tertiary referral center; an Iranian experience
}

Sousan Rasooli ${ }^{1}$, Farnaz Moslemi ${ }^{2 *}$

1. Department of Anesthesiology, Alzahra Teaching Hospital, School of Medicine, Tabriz University of Medical Sciences, Tabriz, Iran

2. Medical Education Research Center, Tabriz University of Medical Sciences, Tabriz, Iran

\begin{abstract}
Objective: The aim of the present study was to evaluate the obstetric admissions to the intensive care unit (ICU) in the setting of a tertiary referral hospital in an attempt to identify the risk factors influencing maternal outcome.

Method: In a prospective, cross-sectional study, all parturient patients during pregnancy and up to 6 weeks postpartum admitted to the ICU of a tertiary referral hospital between 2013 and 2014 were evaluated. Demographic data, medical histories, pregnancy, and intrapartum and postpartum data were collected. Moreover, interventions and fetomaternal outcomes were noted.

Results: Ninety nine obstetric patients were admitted to the ICU. Fifty seven percent of the admissions were postpartum. The main indications for admission were hypertensive disorders $(37.3 \%)$, and obstetric hemorrhage (13.1\%). Non-obstetric indications of ICU admission were the cardiac diseases.

Conclusion: The major obstetric indications for admission in our study were hypertensive disorders of pregnancy and obstetric hemorrhage.
\end{abstract}

Keywords: Pregnancy; Intensive care unit; maternal mortality; morbidity

\section{Introduction}

Although maternal mortality rates have greatly fallen over the last few decades because of improvements in obstetric care, maternal morbidity remains to be a challenge in the developing world. Thus maternal mortality is an inadequate measure of the quality of obstetric

care success in the developing countries. Therefore, attention has been shifted to alternative indicators of care such as severe maternal morbidity [1,2].

Obstetric patients are particularly unique cases for intensive care unit (ICU) physicians;

\footnotetext{
Corresponding author:

Farnaz Moslemi, MD

Department of Anesthesiology, Alzahra Teaching Hospital, Tabriz University of Medical Sciences, Tabriz, Iran E-mail: moslemi_fa@yahoo.com Tel: +989141130034 Fax: +984135566449
}

Receive date: 2014-08-17| Accept date: 2014-09-01| Publish date: 2014-09-21

DOI: 10.7575/aiac.abcmed.15.03.02.08 
they are young and healthy, and in reproductive years, their management is challenged by concerns for fetal viability, altered maternal physiology, and diseases specific to pregnancy $[3,4]$.

Increasing numbers of women with underlying chronic medical conditions are now able to conceive and carry a fetus to term. Women with longstanding diabetes mellitus or complex congenital cardiac defects, specially require a greater degree of medical care throughout the antepartum and postpartum period, and may require a period of observation in an ICU throughout labor and delivery $[3,5]$. The present study was conducted to evaluate the obstetric admissions to the ICU in the setting of a tertiary referral hospital in an attempt to identify the risk factors influencing maternal outcome.

\section{Method}

The present study was approved by the Local Ethics Committee at the Alzahra Hospital, Tabriz University of Medical Sciences, Tabriz, Iran. A prospective, observational hospitalbased study was conducted on all the obstetric patients admitted to the obstetric ICU in the Alzahra Hospital, a tertiary referral universityaffiliated hospital, between April 2013 and April 2014.

The obstetric patients seeking care for delivery at the medical center are admitted to the Department of Obstetrics for delivery after initial evaluation at the emergency department. After delivery, those patients requiring intensive care because of a postpartum cause complicating the delivery are admitted to the ICU, where the intensive care medicine specialists assume the primary responsibility. The adult ICU beds are served by three attending anesthesiologists specialized in intensive care medicine and rotating anesthesiology residents.
For the purpose of the present study, one year prospective review of all the obstetric admissions (antenatal and postnatal up to 6 weeks post delivery) was done. Total numbers of obstetric patients admitted to ICU, their demographic details, medical and obstetric history, the preexisting medical disorders, causes of admission, interventions, duration of stay and fetomaternal outcomes were listed. Admission criteria in the obstetric ICU were critically ill obstetric patients requiring ventilator support or major organ supportive therapy.

Data were presented as mean \pm standard deviation (SD), or frequency (percentage). The collected data were evaluated with Statistical Package for Social Sciences, version 17.0 (SPSS, Chicago, Illinois).

\section{Results}

During the study period, 99 obstetric ICU admissions were recorded. The mean maternal age of the patients was $28.4 \pm 4.9$ years. Duration of ICU stay ranged from 2 to 11 days. Eighty nine (89.8\%) patients were discharged with good condition, but nine $(9 \%)$ patients were transferred to a general hospital for further treatment. The baseline characteristics of the patients at admission are shown in Table 1.

Pregnancy-related hypertensive disorders were the most common obstetric causes of admission (29 patients, 29.2\%). Two patients were admitted to the ICU due to pulmonary edema requiring ventilatory support. Five patients were admitted with eclamptic seizures. Two of these patients were subsequently diagnosed with posterior reversible encephalopathy syndrome after brain magnetic resonance imaging. Clinical characteristics of cases admitted to obstetric ICU are listed in Table 2. In this study hemorrhage was the second leading cause of obstetric admissions to the ICU. Severe 


\begin{tabular}{|c|c|}
\hline Maternal age (years) & $28.4 \pm 4.9$ \\
\hline \multicolumn{2}{|l|}{ Time of admission to } \\
\hline hospital, n (\%) & $42(42.4 \%)$ \\
\hline Antepartum & $53(53.5 \%)$ \\
\hline Postpartum & $4(4 \%)$ \\
\hline \multicolumn{2}{|l|}{ Postabortal } \\
\hline $\begin{array}{l}\text { Direct hospital admission, } n \\
(\%)\end{array}$ & $63(63.6 \%)$ \\
\hline $\begin{array}{l}\text { Referred from other centers, } \\
\text { n }(\%)\end{array}$ & $37(37.3 \%)$ \\
\hline \multirow{3}{*}{\multicolumn{2}{|c|}{$\begin{array}{l}\text { Maternal control during } \\
\text { pregnancy, } \mathrm{n}(\%) \\
\text { Good } \\
\text { Not good }\end{array}$}} \\
\hline & \\
\hline & \\
\hline Gestational age (weeks) & $32.1 \pm 1$ \\
\hline \multicolumn{2}{|l|}{ Mode of delivery, $\mathrm{n}(\%)$} \\
\hline Vaginal delivery & $27(27.2 \%)$ \\
\hline Cesarean section & $72(72.2 \%)$ \\
\hline
\end{tabular}

Table 1: Demographic data of patients

hemorrhage might occur as a result of uterine atony, uterine rupture, placenta previa, and retained placenta (Table 2). Postpartum sepsis duo to chorioamnionitis was the basis of ICU admission in two patients. Of these patients, one case was hemodynamically unstable and required vasopressor support. Cardiac diseases were the major non-obstetric and coexisting causes of maternal admission to the ICU (13 patients, $13.1 \%$, Table 2 ).

In the study period, there was one maternal death due to disseminated intravascular coagulation and multi-organ failure following septic shock. The specific interventions made in the ICU are shown in Table 3.

\section{Discussion}

Pregnancy, delivery and postpartum can be complicated by severe maternal morbidity necessitating ICU admission. Management of critically ill obstetric patients is very complex and requires cooperation among obstetrician, interventionist and anesthesiologist $[3,6]$.

The data presented in this study represents an overview of the spectrum of complications associated with pregnancy in a tertiary care hospital. The major obstetric indications for admission in our study were hypertensive disorders of pregnancy and obstetric hemorrhage. This finding is in according with the findings of previous reports $[2,7,8]$. It has been emphasized that early detection and prompt referral to tertiary centers with intensive care facilities to provide optimum care of circulation, blood pressure and ventilation could minimize the prevalence of multi-organ failure and mortality in critically ill obstetric patients [2,9]. Aggressive blood pressure control is an important end point in the treatment of these young patients in whom acute increases of blood pressure may pose significant risk. Therapy can be given by continuous or bolus infusion, which ideally requires direct measurement of intra-arterial blood pressure to rapidly achieve end points while avoiding excessive decrease in the blood pressure.

A significant number of maternal deaths in preeclampsia are related to pulmonary edema. Hemodynamic monitoring using central venous pressure data in severe preeclampsia is unreliable and should not be used. In this study, invasive monitoring of blood pressure was performed with arterial line and central venous catheter. In the present study, hemorrhage (uterine atony, placenta previa and placenta abruptia) was the second cause of admission to the ICU. This finding is consistent with that of the previous review by Zeeman [3]. Similar to the findings of Selo-Ojeme and colleagues, our study showed that emergency caesarean delivery increased the risk of admission to the ICU [2]. Furthermore, Panchal et al revealed that emergency cesarean section 
Number (\%)

\section{Obstetric diseases}

$57(57.5 \%)$

Hypertensive disorders

Severe preeclampsia

Eclampsia

$29(29.2 \%)$

$2(2 \%)$

HELLP syndrome

$5(5 \%)$

Ante- and postpartum hemorrhage

Placenta previa

$6(6 \%)$

Placenta abruptia

$2(2 \%)$

Uterine rupture

$2(2 \%)$

Uterine atony

$2(2 \%)$

\section{Preexisting non-obstetric diseases}

\section{Hematologic}

ITP

$2(2 \%)$

Factor IV deficiency

$2(2 \%)$

\section{Heart disease}

Valvular heart disease (rheumatic)

$6(6 \%)$

Congenital heart disease

$2(2 \%)$

Peripartum cardiomyopathy

$3(3 \%)$

Arrhythmias

$2(2 \%)$

\section{Endocrine disease}

Diabetes mellitus

Hypothyroidism

\section{Autoimmune disease}

Systemic lupus erythematosus

Myasthenia gravis

Nephritic syndrome

Respiratory disease

Asthma

$5(5 \%)$

$2(2 \%)$

Pulmonary embolism

Liver disease

Hepatic cirrhosis

Hepatitis

Budd-Chiari syndrome

Neurologic disease

Epilepsy

Brain tumors

Infectious disease

Chorioamnionitis

Trauma

Abdominal trauma

$2(2 \%)$

$1(1 \%)$

$1(1 \%)$

$1(1 \%)$

$2(2 \%)$

$3(3 \%)$

$1(1 \%)$

$1(1 \%)$

$1(1 \%)$

$2(2 \%)$

$3(3 \%)$

$1(1 \%)$

Table 2: Clinical characteristics of cases admitted to obstetric ICU and postpartum hemorrhage increased the risk of admission to the ICU [10].

\begin{tabular}{lc}
\hline \multicolumn{1}{c}{ Interventions } & $\begin{array}{c}\text { Number of } \\
\text { patients (\%) }\end{array}$ \\
\hline Mechanical ventilation & $5(5 \%)$ \\
\hline Antihypertensive therapy & $32(32.3 \%)$ \\
\hline Inotropic support & $9(9 \%)$ \\
\hline Arterial line insertion & $35(35.3 \%)$ \\
\hline $\begin{array}{l}\text { Central venous catheter } \\
\text { placement }\end{array}$ & $21(21.2 \%)$ \\
\hline Echocardiography & $46(46.4 \%)$ \\
\hline Abdominal ultrasound & $38(38.3 \%)$ \\
\hline Brain CT & $11(11.1 \%)$ \\
\hline Brain MRI & $9(9 \%)$ \\
\hline CT angiography & $13(13.1 \%)$
\end{tabular}

Table 3: Interventions made in obstetric patients admitted to ICU

\section{Conclusion}

Hypertension-related complications and massive obstetric hemorrhage in previously low-risk women are the leading causes of obstetric patients' admission to the ICU. The admission rate to ICU and the problems faced by critically ill parturient patients may be reduced by improving the management of hypertensive disease during pregnancy and by reducing the prevalence of hemorrhagic complications through emphasis on the early detection and anticipation. When complications arise early, intervention and treatment on a multidisciplinary basis including ICU admission for ventilatory support, invasive monitoring, and vasoactive drug infusions, can alleviate progression of organ dysfunction and improve prognosis. 


\section{References}

1. Baskett TF. Epidemiology of obstetric critical care. Best Pract Res Clin Obstet Gynaecol. 2008;22:763-774.

2. Selo-Ojeme DO, Omosaiye M, Battacharjee P, Kadir RA. Risk factors for obstetric admissions to the intensive care unit in a tertiary hospital: a case-control study. Arch Gynecol Obstet. 2005; 272:207-210.

3. Zeeman GG. Obstetric critical care: a blueprint for improved outcomes. Crit Care Med. 2006;34:S208-S214.

4. Ashraf N, Mishra SK, Kundra P, Veena P, Soundaraghavan S, Habeebullah S. Obstetric patients requiring intensive care: a one year retrospective study in a tertiary care institute in India. Anesthesiol Res Pract. 2014;2014:789450.

5. Devabhaktuni P, Samavedam S, Thota G, Pusala S, Velaga K, Bommakanti L, Nawinne M, Thomas P. Clinical profile and outcome of obstetric ICU patients. APACHE II, SOFA, SAPS II and MPM scoring systems for prediction of prognosis. Open J Obstet Gynecol. 2013;3:41-50.

6. Togal T, Yucel N, Gedik E, Gulhas N, Toprak HI, Ersoy MO. Obstetric admissions to the intensive care unit in a tertiary referral hospital. J Crit Care. 2010;25:628-633.

7. Gilbert TT, Smulian JC, Martin AA, Ananth CV, Scorza W, Scardella AT; Critical Care Obstetric Team. Obstetric admissions to the intensive care unit: outcomes and severity of illness. Obstet Gynecol. 2003;102:897-903. 8. Waterstone $\mathrm{M}$, Bewley S, Wolfe $\mathrm{C}$. Incidence and predictors of severe obstetric morbidity: case-control study. BMJ. 2001;322:1089-1093.

9. Gupta S, Naithani U, Doshi V, Bhargava V, Vijay BS. Obstetric critical care: A prospective analysis of clinical characteristics, predictability, and fetomaternal outcome in a new dedicated obstetric intensive care unit. Indian J Anaesth. 2011;55:146-153.

10. Panchal S, Arria AM, Harris AP. Intensive care utilization during hospital admission for delivery: prevalence, risk factors, and outcomes in a statewide population. Anesthesiology. 2000;92:1537-1544. 